\title{
Microcomputer display timing: Problems and solutions
}

\author{
ADAM V. REED \\ New School for Social Research, New York, New York 10003
}

\begin{abstract}
The 525-line composite video display standard is described and discussed from the viewpoint of its usefulness in psychology experiments. While a frame is displayed every $33.3 \mathrm{msec}$, it consists of two interlaced 262.5-line subframes displayed in $16.7 \mathrm{msec}$ each. Thus, a display consisting of 16 or fewer video lines may be presented in 1 msec or less. The hardware of some microcomputers may be modified to make the video vertical synchronization timing signal available to latency timing software. This modification permits response signal and latency timing with 1-msec or better precision. A modification that permits display blanking under software control is also noted, and a detailed description of both modifications is given for the popular APPLE II microcomputer. Other problems discussed include moving display materials into display buffer areas in time for single-scan precision timing, software speed problems, and the tradeoffs between video and light-emitting diode displays.
\end{abstract}

Although most low-cost microcomputer systems are designed for game playing and other applications that do not ordinarily require precision timing, they can provide cost-effective laboratory display control. With minor hardware changes and appropriate software, they can equal the performance of far more expensive systems sold specifically for laboratory applications. Video displays may be used without loss of millisecond precision, provided one is willing to: (1) learn how they work, (2) perform minor modifications of the computer's electronic hardware, and (3) part with a small portion of the design flexibility possible with traditional tachistoscopes and expensive point-plot display systems. If the researcher's stimuli permit the use of light-emitting diode (LED) instead of cathode-ray tube (CRT) screens, Requirements 2 and 3 become optional. Money for LEDs and some time spent writing software are often all one needs.

\section{AMERICAN VIDEO DISPLAY STANDARD}

Video display standards differ from country to country. Scan pattern and display frequency differences, as well as differences in video bandwidth and in the number of lines in the display, combine to account for 14 different national and regional standards. The present discussion will apply in detail only in Canada, Japan, the United States, and other countries using the North American/East Asian video standard. Researchers elsewhere should consult locally to determine locally applicable video display standards.

The 525 lines of the American video standard include 524 whole lines (Lines 1-524) plus two half-lines: the right half of Line 0 and the left half of Line 525. Evenand odd-numbered lines are scanned in an interlaced pattern in alternating frames. Sixty frames are presented per second; this corresponds to the 30 pairs of frames necessary to display the entire complement of 525 lines. The scan of each line proceeds from left to right and slightly downward; the even- or odd-numbered lines are scanned in succession from the top to the bottom of the screen. Even-numbered frame scans start at the top middle of the screen and end in the bottom right-hand corner; odd-numbered frame scans start in the top left corner and end in the middle of the bottom line.

Only the most expensive memory-mapped displays, such as the MATROX high-resolution video boards available for some microcomputer systems, use the interlace feature of the video standard. Even those displays seldom use the full 525 lines: The MATROX high-resolution boards, for example, use the center 512. Most microcomputer video displays use 258 or fewer lines. (The very popular APPLE II, for example, uses only the center 192 lines.) The same lines are output 60 times/sec, so that each line output by the com. puter actually occupies two adjacent lines in the 525 . line standard. Each line in such a display moves up and down slightly between successive frames. A line shown for only one frame is only half as wide vertically as a line shown for two frames if the video monitor beam is perfectly focused. In practice the focus is likely to be less than perfect, making the actual ratio of vertical widths larger than the theoretical .5 to 1 .

A total of 16 video lines can be shown under this standard in a single millisecond. Once the modifications discussed below have been made, any display less than 16 video lines high (e.g., 2 lines of text) can be presented with better than millisecond precision. Thus, if we wish to present a response signal with a specific time lag after the presentation of visual information, or if we wish to record in milliseconds the latency of response from presentation, we can do so, provided we know 
when the stimulus is first displayed. To know this, we must know with some precision where on the screen the video beam is writing at any given moment. The easiest way to do this is to synchronize the experiment control program with the videoscan.

\section{MODIFICATION 1: VERTICAL SYNCH MONITORING CIRCUIT}

The composite video signal that the computer feeds into the video monitor is composed of brightness pulses, which are positive with respect to baseline, and synchronization ("synch") pulses, which are negative-going. The synch pulses are of two kinds, wide for vertical synch and narrow for horizontal synch. The vertical synch pulse occurs between display frames, and the time at which any point on the screen is displayed can be readily calculated relative to that pulse. The size of these pulses is about $1 \mathrm{~V}$, which is too low to activate most digital sensing circuits. Fortunately for the experimenter, the synch pulses are generated digitally in the microcomputer before being combined with video information to form composite video. In general, it is not a good idea to try to sense the digital vertical synch pulse directly: The composite video signal is generated by a delicately balanced resistor network, and any load on an input into that network may affect its balance. However, the timing for the vertical synch pulse is also generated internally, and this timing signal may be sensed without affecting the balance of the composite video signal.

Thus, the one hardware modification necessary to use any popular microcomputer in the psychology laboratory is to connect the vertical synch pulse timing signal to one of the sense lines that can be tested from software. The first step is to identify the vertical synch pulse timing signal in the microcomputer's circuit diagram. In the APPLE II this signal is called V4, and it is available to Pin 12 of the integrated circuit (IC) socket at D11 and at Pin 10 of IC socket B11. Of the three digital sense switches available to the user, SW0 and SW1 are normally assigned to subject-response buttons, leaving SW2 at Pin 4 of socket J14 for sensing the vertical synch timing signal. Multiplexed encoding of this signal with subject-response buttons should be avoided, since a spurious code is produced whenever vertical synch and a response overlap in time. The connection is best implemented by wire wrapping a connecting lead to the appropriate pins of two wirewrap sockets. The IC at B11 and the connector at J14 are then removed, and the connected wire-wrap sockets are inserted in the on-board sockets of the APPLE II. Then the IC at B11 and the connector at $\mathrm{J} 14$ are plugged into the wire-wrap sockets. The signal connection at B11 rather than the one at D11 is used to avoid incompatibility with plug-in peripheral boards.

\section{MODIFICATION 2: DISPLAY-BLANKING CIRCUIT}

It is often useful, and sometimes essential, to be able to suppress the display of all or part of a screen without changing the content of display memory. Fortunately, a display-blanking circuit may be built for almost any microcomputer system with digital outputs. The dots composing the video display are usually kept in register by means of a clocked circuit, such as a 7474 or equivalent Type D flip-flop, or a 7496 or equivalent shift register. Such circuits tend to have "ENABLE/CLEAR" input lines (e.g., Pin 1 on a 7474 flip-flop A or Pin 16 on a 7496). When this line is high, the circuit operates normally; when it is low, the output of the clocked circuit is held at zero. Input is normally pulled up to $+5 \mathrm{~V}$ on board. A blanking circuit may be implemented by disconnecting this line from $+5 \mathrm{~V}$ and connecting it to a software-controlled digital output line.

On the APPLE II, this modification may be implemented by tying Output Line AN\#3, at Pin 12 of J14, to Pin 1 of the 74LS74 at B10. The wire-wrap socket technique used for Modification 1 may be used, with one change: After the wire-wrap connection to Pin 1 of the B10 wire-wrap socket is made, the pin must be cut to half its original length, to disconnect it from the on-board $+5-V$ pull-up.

\section{DISPLAY MEMORY ACCESS HARDWARE}

Three types of access to display memory have been used in microcomputer display hardware: serial, register, and direct memory mapping.

\section{Serial Access}

Early microcomputer displays used the serial access method, and some display boards of this type are still being sold to unwary users. The problem with serial (current loop or RS232) access is speed, or rather, the lack of speed. The XITEX SCT-100, a typical serially accessed board, can change displayed characters at a maximum rate of 30 characters/sec ( 300 baud). That means one character per two videoscans. Changing a full screen takes more than $30 \mathrm{sec}$. Even the fastest serial board, the MATROX MTX with serial interface, can change characters only at a rate of 9,600 baud (i.e., 16 characters/scan). Few of the serially accessed boards have more than one display page; those that do provide external access only to the page that is currently being displayed.

A blanking circuit is essential when serially accessed displays are used in psychological experiments. The slower boards, such as the XITEX SCT-100, may only be used for those experiments in which masking or other rapid succession of multiline displayed material is not required. For typical experiments on memory 
or decision making, the single-display screen may be filled while blanked out, then unblanked from one videoscan to the next. This procedure controls stimulus onset time, and the screen may be blanked again at stimulus offset. True control of stimulus duration, via masking, can only be achieved for one-line displays. In this application, the mask is placed on the line below the to-be-masked stimulus ahead of time, and the cursor is moved to the bottom of the screen. The blanking circuit is used to display only a part of the screen, including the stimulus line but not the mask. A precisely timed line-feed character causes the display to scroll at the desired time, placing the mask where the stimulus used to be, from one scan to the next. The blanking circuit is then used to display the mask but not the old stimulus line, which is then the next line up.

The above technique may be used to mask larger displays on faster serial boards. On the serially interfaced MATROXMTX, displays as large as 12 80 -character lines may be masked by scrolling from one scan to the next. Serially interfaced boards with multipledisplay screens in memory may have all of the displays loaded while the screen is blanked, thus permitting full-screen masking.

Serially interfaced video boards are sometimes bought by mistake. Computer salesmen have been known to tell customers that the XITEX SCT-100 is an S-100 plug-in, and customers have bought the board believing that its video memory is directly addressable, via the S-100 bus, by their microprocessors. The SCT100 does plug into the S-100 connector, but the only S-100 lines from which it can receive anything are those carrying unregulated dc power.

\section{Register Access}

This method is used in most plug-in graphic boards, including XEDAX GRAPHIC and MATROX 256 and 512 series. Although advertised as "videorams," the display memories of those boards are not directly addressable by the processor. Instead, the display memory is accessed through three memory locations that serve as registers. To change a dot on the screen, it is necessary to place its $x$ location in one register, its $y$ location in a second, and the direction of the desired change (on or off) in a third. Only then does the desired change take place. A direct memory-mapped high-resolution graphic display, such as the one incorporated into the APPLE II, can change up to seven display dots with a single memory storage operation. A microcomputer system with a register access display board needs 21 storage operations to do as much. While actual performance depends on processor speed and instruction set, it makes sense to calculate the number of dots a board can change in the $16 \mathrm{msec}$ between scans before deciding to buy it.

\section{Direct Memory Mapping}

Direct memory mapping means that every character in an alphanumeric or low-resolution graphic display and every group of dots in a high-resolution graphic display can be directly addressed by the processor as 1 byte in its working memory space. With this technology it is possible to transfer from one-third to a whole screen of alphanumeric or graphic information from a "ghost" location into the display area between one screen scan and the next. The same can be done with up to oneeighth of a dot-oriented high-resolution screen. Actual performance depends on the method of sharing the memory between the display and the processor. Four common methods are timesharing, multiplexing, cycle stealing, and mutually transparent access.

\section{Timesharing}

Timesharing, used in the minimum-cost PIAI display board for the minimum-cost KIM-1 microcomputer, employs the processor for display control. Thus, the microprocessor is timeshared between display control and other operations, such as changing the content of display memory. The latter can be performed only during horizontal and vertical retrace parts of the videoscan cycle: about $40 \%$ of the time. This means that only one-third of a character-oriented screen can be changed from one videoscan to the next.

\section{Multiplexing}

Multiplexing, used in the Commodore PET and CBM 2001 series microcomputers, outputs the display memory to the screen whenever that memory is not being accessed by the processor. If display memory is accessed at times other than the retrace sections of the videoscan cycle, the display blinks perceptibly. However, the processor is available for operations other than display memory access during the display portions of the video cycle. If a display-blanking circuit is installed, up to two-thirds of a character-oriented screen may be transferred into the display area from one scan to the next.

\section{Cycle Stealing}

Cycle stealing, used in microcomputer systems with processors operating at speeds above $2 \mathrm{MHz}$, means that display operations are performed during alternate processor cycles. Display operations take place while the processor is placed in a special state that suspends memory access ("wait") while permitting other operations internal to the processor. The advantage of this method is that the processor cannot interfere with display operations while operating almost normally. The disadvantage is the almost: Display operations may cause unpredictable perturbations in the speed of program execution.

\section{Transparent Access}

Transparent access, the technique used in MATROX alphanumeric video RAM boards and in the APPLE II built-in video system, is a recent innovation made 
possible by very high-speed memory chips. In this technique, each processor cycle is split into two memory access cycles, one of which can be used only by the processor and the other, only by the display circuitry. With this technique, the processor functions as though the display were not there, and vice versa. Transparent access permits the transfer of a whole screen of character information from a ghost area into a display area from one videoscan to the next. As long as display durations in multiples of $16.7 \mathrm{msec}$ are acceptable, a microcomputer with transparent access video can serve as a whole-screen $\mathrm{n}$-field tachistoscope, with $\mathrm{n}$ limited only by the total amount of memory installed in the system.

Transparent access video displays seem almost perfectly suited for most psychological experimentation, and now that they are available in relatively low-cost systems, it makes little sense for a psychological laboratory to buy any other kind. For this reason, the discussion of software problems below is oriented primarily toward displays of this type.

\section{DISPLAY SOFTWARE}

Consider, as an example, the following display sequence: The original content of the screen, a fixation frame, is replaced by a frame containing a word, which stays on for two scans $(33.3 \mathrm{msec})$ and then is replaced by a mask. The job is easiest on microcomputers in which the segment of memory being displayed, or display page, may start in any of several locations in the computer's memory. Before the trial, the fixation frame, the frame with the word, and the frame with the mask are placed in three of the available display pages. When we wish to display the word, we wait for the next vertical synch pulse, or its timing signal, and then place the origin of the word display page in the "current display page origin" register. We then wait for the second onset of the vertical synch signal, which occurs $33.3 \mathrm{msec}$ later, and place the value of the mask page origin in that register.

The job is somewhat more difficult in microcomputers with only two valid display pages. Thus, if we start with the blank frame in Display Page 1 (currently being displayed) and the word frame in Display Page 2, the mask frame will have to reside in a "ghost" page elsewhere in memory. As before, we wait for a vertical synch pulse and then switch the display from Display Page 1 to Display Page 2. We now have $33 \mathrm{msec}$ in which to transfer the mask frame from its ghost display page into Display Page 1.

If the word is to be shown for only one scan, we would have $16 \mathrm{msec}$ for the transfer to be done in one piece. Another $16 \mathrm{msec}$ could be added to these times by beginning the transfer earlier: Soon after the beginning of the last scan of the empty frame, we can begin replacing the content of Display Page 1 locations that have already been scanned. Another $16 \mathrm{msec}$ can be added by continuing with the last part of the transfer after switching back from Display Page 2 to Page 1 . We need only make sure that the scan process does not catch up with us before the transfer is completed. Thus, even if a decision is made to transfer a whole page, there are $48 \mathrm{msec}$ to do it in. We may also decide to transfer only those locations in which the content of the ghost page with the mask frame and of Display Page 1 with the empty frame differ. This takes less time than transferring the whole page.

Some microcomputers have only one valid display page origin. In these computers, a two-scan display duration requires that a page be transferred, even in two pieces, in $33 \mathrm{msec}$ or less; a single-scan display would require $24-\mathrm{msec}$ page transfer. These times seem quite adequate until one remembers that displays take up a lot of memory space. A typical text or low-resolution display page might contain $1 \mathrm{~K}(1,024)$ bytes of memory. A high-resolution display page in the APPLE II contains $8 \mathrm{~K}$ bytes. Thus, even the transfer of only the central one-eighth of a page means transferring $1 \mathrm{~K}$ bytes. If the MOVE routine of the computer's monitor-in-ROM is used for this transfer, it takes over $50 \mathrm{msec}$. Indeed, using monitor routines or an interpreted language such as BASIC is totally out of the question when milliseconds count. Linear assembler code must be written, and it must exploit every available feature of the microprocessor at the experimenter's disposal. The 6502, for example, incorporates powerful addressing modes that can be used for real-time operations. A table of source and destination base addresses for each line can be set up ahead of time, and then the indirect indexed addressing mode can be used to transfer blocks of data. When this is done, a 1K-byte transfer can be performed in less than 14 msec.

\section{LED DISPLAYS}

When display durations must be graded in increments finer than the $16.7 \mathrm{msec}$ mandated by video monitors, microcomputers may be interfaced to LED displays. Some of the least expensive microcomputers, such as the KIM, SYM, and the AIM-65, incorporate LEDs in their designs as sold. LEDs are fast: They can be switched on or off in a fraction of a microsecond. They are limited in the variety of displays they can generate, since the KIM and the SYM each have six-character eight-segment displays capable of generating numbers and a few of the letters. The AIM-65 has a 20-character display capable of showing numbers, uppercase letters, and even some symbols. Larger and more flexible displays can be built and readily interfaced to microcomputers, but such displays are expensive. An eightcharacter LED module capable of displaying upper- 
and lowercase characters currently sells for about $\$ 40$. Thus, the LED equivalent of three 40-character lines, the maximum displayable in under $1.5 \mathrm{msec}$ on a video monitor, costs $\$ 600$, the price of four video monitors.

As far as timing is concerned, the only possible trap for the LED user is the monitor software supplied in ROM with LED-based microcomputers. Although the LEDs themselves are fast, the software often is not. The monitor software on the KIM, for example, goes into a $.5-\mathrm{msec}$ loop when displaying each digit, and the complete six-digit display takes nearly $4 \mathrm{msec}$. But the experimenter who writes her or his own software can certainly enjoy the convenience of computer control without sacrificing exact timing essential for reliable experimentation.

(Received for publication May 11, 1979; revision accepted September 29, 1979.) 